\title{
WOOD DEGRADING MUSHROOMS POTENTIALLY STRONG TOWARDS LACCASE BIOSYNTHESIS IN PAKISTAN
}

\author{
Zill-E-Huma Aftab, Shakil Ahmed \\ University of The Punjab \\ Pakistan \\ Arusa Aftab \\ Lahore College for Women University \\ Pakistan \\ IfFAT SidDique \\ Eastern Cereal and Oilseed Research Centre \\ Canada \\ Muzammil Aftab \\ Government College University \\ Pakistan \\ Zubaida Yousaf \\ Lahore College for Women University \\ PAKISTAN \\ Farman Ahmed Chaudhry \\ Minhaj University \\ PAKISTAN \\ (Received November 20ig)
}

\begin{abstract}
In present study, Pleurotus ostreatus, Ganoderma lucidum, Ganoderma ahmadii, Ganoderma applanatum, Ganoderma australe, Ganoderma colossus, Ganoderma flexipes, Ganoderma resinaceum, Ganoderma tornatum, Trametes hirsutus, Trametes proteus, Trametes pubescens, Trametes tephroleucus, Trametes versicolor, Trametes insularis, Fomes fomentarius, Fomes scruposus, Fomitopsis semitostus, Fomes lividus, Fomes linteus, Phellinus allardii, Phellinus badius, Phellinus callimorphus, Phellinus caryophylli, Phellinus pini, Phellinus torulosus, Poria ravenalae, Poria versipora, Poria paradoxa, Poria latemarginata, Heterobasidion insulare, Schizophyllum commune, Schizophyllum radiatum,
\end{abstract}


Daldinia sp., Xylaria sp., were collected, isolated, identified and then screened qualitatively for their laccase activity. Among all the collected and tested fungi Pleurotus ostreatus 008 and 016, Ganoderma lucidum 101,102 and 104 were highly efficient in terms of laccase production. The potent strains were further subjected to Quantitative laccase bioassay for partial purification and characterization of industrially important enzyme.

KEYWORDS: Laccase, lignin degradation, qualitative screening, white rot fungi, wood degradation.

\section{INTRODUCTION}

Lignocellulosic biomass is mainly composed of three major components, which are cellulose, hemicelluloses and lignin ( $\mathrm{Li}$ et al. 2019). Laccases (E.C, 1.10.3.2 benzenediol: oxygenoxidoreductases) belonging to multicopper oxidase family (blue oxidases) are the most important among the lignolytic complex primarily responsible for decomposition of lignin, most widely distributed natural polymer (Baldrian 2006, Pažitný et al. 2019). Yoshida first discovered the Laccases in 1883, after working on latex from the plant Japanese lacquer (Rhus vernicifera) which hardened when exposed to air (Gianfreda et al. 1999). So the laccases scored the position among the oldest enzymes. Lignin is hard to digest by animal enzymes but during secondary metabolism active laccases have been studied in plants, some insects, (Dittmer et al. 2009) a few bacteria (Claus 2004) and lots of fungi (Kumaran et al. 2011).

Fungal classes Ascomycetes, Deutromycetes and particularly Basidiomycetes exhibit both extra as well as intracellular laccase activity. However, extracellularly produced amount of enzyme is much higher. Ascomycetous fungi showing potential to degrade lignin include Aspergillus sp., Geotrichum sp., Oxyporus latemarginatus, Trichoderma atroviride, Trichoderma harzianum, Trichoderma longibrachiatum (Dhouib et al. 2005). Among Basidiomycetes species Trametes versicolor and Phanerchaete chrysosporium are till now the most worked organism for laccase synthesis because of their exceptional lignin degrading capacity. Examples of other laccase producers include Pleurotus ostreatus (Hou et al. 2004), Marasmius quercophilus (Tagger et al. 1998), Pleurotus pulmonarius (Souza et al. 2002), Ganoderma adspersum (Songulashvili et al. 2007), Pycnoporus cinnabarinus and Pycnoporus sanguineus (Eggert et el. 1996, Pointing and Vrijmoed 2000), Chaetomium thermophilium (Chefetz et al. 1998) and Phelbia radiata and P. floridensis (Arora and Gill 2012, Lundell et al. 1990).

Objective of the present study was to evaluate the biosynthetic potential of the local strains.

\section{MATERIALS AND METHODS}

Field surveys were conducted from the months of May to September for the collection of wood rotting fungi. Visits were conducted to different natural and manmade forest areas of Lahore (Quaid-e-Azam Campus, Punjab University, Jinnah Garden, Lahore Safari Zoo, Jallo Mor Park), Chhanga Manga (Jheel side, Wood Toll, Interior of Jungle, Village side) Bhai Pheru (Head Ballocki), Sialkot, Gujrat, Gujranwala, Khan's Pur (Jhica Gully, Chhangla Gully, Ayub National Park, Khan's Pur Campus, Punjab University) Gilgit (Ghizar, Phander, Astoor), Chitral (Kelash) Swat (Kalam, Malakund, Malam Jabba, Mutlatan, Mengora) Bisham, Chillas, Azad Kashmir (Neelam Velly, Muzaffar Abad) Narran, Kaghan, Shugran. Most of the samples were collected form decayed wood but some collection was also done from living trees. 
The isolation of fungi was done on 2\% MEA (Malt Extract Agar) medium at $\mathrm{pH}$ 6. Before inoculation collected samples were surface sterilized with $(15 \% \mathrm{v} / \mathrm{v}) \mathrm{H} 2 \mathrm{O} 2$ solution. Identification of isolated fungi was done on the basis of morphological (colour, texture, appearance, and diameter of colonies) and microscopic (microstructures) characteristics as well as on the basis of their fruiting bodies collected, following the keys developed by (Selvam et al. 2012, Gonthier and Nicolotti 2007, Luley 2005). Pure cultures were used for further species identification.

Plate screening was done by following More et al. (2011) to identify potent laccase producing wood rotting fungi in extracellular fluid. ABTS (2, 2'-azino-bis-3-ethylbenzothiazoline-6sulfonic acid (biochemical reagent) $0.5 \mathrm{mM}$ per $\mathrm{ml}$ in sodium acetate buffer $(\mathrm{pH} 4.5,0.1 \mathrm{M}$ ) was added in the medium. Guaiacol $(2 \mathrm{mM})$ in acetate buffer $(10 \mathrm{mM}, \mathrm{pH} 5.0)$ was added in the other set of media for laccase assay. Screening was done at three different $\mathrm{pH}$ levels i.e., 4, 5 and 6. Control was without any oxidizing agent at all the three $\mathrm{pH}$ levels. Whole experimental set up was run in triplicate.

\section{RESULTS AND DISCUSSION}

A total of 96 fungi were isolated from different locations and tested through solid state fermentation assay for qualitative evaluation of laccase using Malt extract agar medium supplemented with two oxidizing agents i.e., guaiacol and ABTS separately incubated at $3 \mathrm{pH}$ levels i.e., 4, 5 and 6. All the strains showed growth on media and oxidized both the substrate as hydrolyzing zone. The results of clear zone were indicated as purple for ABTS assay and yellowish brown with guaicol (Fig. 1).

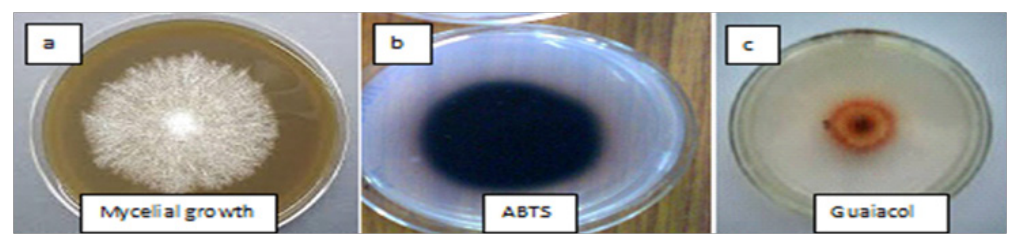

Fig. 1: a) Mycelial growth of wood rotting fungi collected from different locations, b) Laccase assay with ABTS, c) Laccase assay with guaiacol.

The zones of hydrolysis measured for all the screened fungi are given (Tab. 1). Most of the fungi behaved remarkable at $\mathrm{pH} 5$ with ABTS assay. 
Tab. 1: List of identified fungal species and their potential towards Laccase synthesis collected from different locations of Pakistan.

\begin{tabular}{|c|c|c|c|c|c|c|c|}
\hline \multirow{3}{*}{ Fungal species } & \multirow{3}{*}{$\begin{array}{l}\text { Locations of } \\
\text { collection }\end{array}$} & \multicolumn{6}{|c|}{ Zone of hydrolyzation $(\mathrm{mm})$} \\
\hline & & \multicolumn{3}{|c|}{ ABTS } & \multicolumn{3}{|c|}{ Guaiacol } \\
\hline & & $\mathrm{pH} 4$ & $\mathrm{pH} 5$ & pH 6 & pH 4 & pH 5 & pH 6 \\
\hline \multirow{11}{*}{$\begin{array}{l}\text { Pleurotus } \\
\text { osteratus }\end{array}$} & \multirow{7}{*}{ Lahore } & $5.55 \pm 1.32$ & $19.60 \pm 1.29$ & $14.50 \pm 1.45$ & $11.55 \pm 0.76$ & $15.35 \pm 0.11$ & $13.10 \pm 1.08$ \\
\hline & & $10.30 \pm 0.01$ & $16.55 \pm 0.03$ & $11.60 \pm 1.23$ & $12.45 \pm 0.76$ & $13.40 \pm 0.02$ & $10.75 \pm 0.13$ \\
\hline & & $6.00 \pm 0.23$ & $15.45 \pm 1.21$ & $12.25 \pm 0.45$ & $10.40 \pm 0.18$ & $15.55 \pm 0.34$ & $12.25 \pm 0.09$ \\
\hline & & $19.65 \pm 1.23$ & $24.40 \pm 0.97$ & $10.35 \pm 1.56$ & $6.05 \pm 1.01$ & $17.45 \pm 1.09$ & $10.20 \pm 0.87$ \\
\hline & & $10.55 \pm 0.05$ & $27.60 \pm 0.81$ & $7.35 \pm 1.23$ & $10.40 \pm 0.04$ & $15.15 \pm 1.46$ & $13.25 \pm 0.34$ \\
\hline & & $15.55 \pm 1.45$ & $21.05 \pm 0.95$ & $5.75 \pm 0.34$ & $12.00 \pm 1.34$ & $18.45 \pm 0.51$ & $16.95 \pm 0.92$ \\
\hline & & $22.55 \pm 0.52$ & $16.15 \pm 0.01$ & $9.00 \pm 1.01$ & $15.85 \pm 1.09$ & $12.35 \pm 0.56$ & $11.25 \pm 0.81$ \\
\hline & \multirow{3}{*}{$\begin{array}{c}\text { Chhanga Manga } \\
\text { forest }\end{array}$} & $10.55 \pm 0.45$ & $38.30 \pm 0.12$ & $3.65 \pm 1.04$ & $6.55 \pm 0.98$ & $25.10 \pm 0.56$ & $11.75 \pm 1.67$ \\
\hline & & $16.55 \pm 0.63$ & $24.30 \pm 1.39$ & $10.20 \pm 0.05$ & $7.65 \pm 0.45$ & $14.00 \pm 1.05$ & $8.45 \pm 1.69$ \\
\hline & & $13.10 \pm 0.76$ & $14.45 \pm 0.59$ & $16.00 \pm 1.63$ & $12.80 \pm 0.92$ & $15.65 \pm 0.05$ & $7.00 \pm 1.34$ \\
\hline & Bhai pheru & $13.55 \pm 0.65$ & $17.50 \pm 1.38$ & $8.05 \pm 0.36$ & $6.55 \pm 0.02$ & $11.25 \pm 0.17$ & $8.85 \pm 0.18$ \\
\hline & \multirow{4}{*}{$\begin{array}{l}\text { Murre, Khan's } \\
\text { Pur \& Ayubia }\end{array}$} & $11.35 \pm 0.86$ & $25.65 \pm 1.11$ & $10.50 \pm 1.32$ & $12.10 \pm 1.23$ & $21.50 \pm 1.87$ & $11.35 \pm 1.78$ \\
\hline & & $10.00 \pm 0.09$ & $20.55 \pm 0.90$ & $15.70 \pm 0.56$ & $9.65 \pm 0.65$ & $24.15 \pm 0.45$ & $13.35 \pm 0.34$ \\
\hline & & $14.45 \pm 0.54$ & $13.65 \pm 0.04$ & $14.50 \pm 0.14$ & $15.50 \pm 0.24$ & $19.15 \pm 0.34$ & $12.00 \pm 0.44$ \\
\hline & & $10.25 \pm 1.23$ & $17.35 \pm 1.76$ & $10.05 \pm 1.71$ & $10.20 \pm 1.17$ & $24.86 \pm 0.95$ & $11.10 \pm 0.59$ \\
\hline & \multirow{3}{*}{ Sawat } & $10.55 \pm 0.09$ & $41.35 \pm 1.24$ & $11.25 \pm 0.95$ & $10.15 \pm 0.64$ & $39.45 \pm 1.56$ & $13.75 \pm 1.87$ \\
\hline & & $15.45 \pm 0.94$ & $21.55 \pm 0.85$ & $10.45 \pm 0.62$ & $7.45 \pm 0.74$ & $14.65 \pm 0.65$ & $12.75 \pm 0.48$ \\
\hline & & $10.00 \pm 0.76$ & $20.45 \pm 1.45$ & $11.55 \pm 0.43$ & $13.55 \pm 0.51$ & $11.35 \pm 0.67$ & $10.25 \pm 0.76$ \\
\hline & Chitral & $12.35 \pm 0.52$ & $28.55 \pm 0.58$ & $10.35 \pm 0.23$ & $10.00 \pm 0.65$ & $9.75 \pm 0.18$ & $8.95 \pm 0.67$ \\
\hline & \multirow{2}{*}{ Gilgit } & $10.65 \pm 0.76$ & $19.25 \pm 0.01$ & $3.05 \pm 0.65$ & $7.45 \pm 0.18$ & $11.35 \pm 0.87$ & $9.65 \pm 0.43$ \\
\hline & & $10.15 \pm 0.56$ & $19.25 \pm 0.76$ & $11.35 \pm 0.76$ & $15.45 \pm 0.45$ & $24.55 \pm 0.67$ & $13.65 \pm 0.34$ \\
\hline \multirow{8}{*}{$\begin{array}{l}\text { Ganoderma } \\
\text { lucidum }\end{array}$} & \multirow{3}{*}{ Lahore } & $11.75 \pm 0.56$ & $42.85 \pm 0.54$ & $22.95 \pm 0.61$ & $18.00 \pm 0.76$ & $37.05 \pm 1.45$ & $8.00 \pm 0.09$ \\
\hline & & $10.15 \pm 1.65$ & $39.05 \pm 0.92$ & $20.25 \pm 0.75$ & $10.15 \pm 0.23$ & $28.35 \pm 0.96$ & $18.45 \pm 1.54$ \\
\hline & & $15.00 \pm 1.89$ & $23.65 \pm 0.97$ & $10.85 \pm 1.65$ & $11.25 \pm 0.65$ & $12.55 \pm 0.65$ & $10.55 \pm 1.56$ \\
\hline & Chhanga Manga & $10.00 \pm 0.64$ & $38.05 \pm 0.34$ & $15.75 \pm 0.76$ & $10.55 \pm 0.54$ & $37.85 \pm 0.23$ & $14.45 \pm 0.81$ \\
\hline & & $10.95 \pm 0.76$ & $21.05 \pm 0.05$ & $14.35 \pm 0.76$ & $10.85 \pm 0.45$ & $31.15 \pm 0.09$ & $21.65 \pm 1.78$ \\
\hline & & $10.95 \pm 0.97$ & $11.85 \pm 0.76$ & $10.75 \pm 0.57$ & $15.65 \pm 1.34$ & $17.55 \pm 0.86$ & $13.45 \pm 1.98$ \\
\hline & sawat & $13.05 \pm 0.95$ & $23.25 \pm 0.56$ & $11.15 \pm 0.76$ & $10.00 \pm 1.08$ & $9.95 \pm 0.76$ & $8.15 \pm 0.56$ \\
\hline & Khan's Pur & $12.85 \pm 0.76$ & $22.25 \pm 0.49$ & $10.75 \pm 0.85$ & $10.35 \pm 0.63$ & $22.65 \pm 0.98$ & $10.45 \pm 0.04$ \\
\hline \multirow{4}{*}{ G. abmadii } & \multirow{2}{*}{ Lahore } & $7.55 \pm 0.53$ & $11.55 \pm 0.69$ & $13.45 \pm 0.52$ & $15.65 \pm 0.79$ & $23.35 \pm 0.56$ & $13.75 \pm 0.56$ \\
\hline & & $15.25 \pm 0.61$ & $26.85 \pm 0.74$ & $13.15 \pm 0.09$ & $10.95 \pm 0.56$ & $11.00 \pm 1.45$ & $13.05 \pm 0.07$ \\
\hline & Sialkot & $11.95 \pm 0.04$ & $19.15 \pm 0.65$ & $15.85 \pm 0.13$ & $12.25 \pm 0.31$ & $10.75 \pm 0.56$ & $8.35 \pm 0.40$ \\
\hline & Murree & $11.65 \pm 0.78$ & $19.45 \pm 0.32$ & $8.55 \pm 0.21$ & $5.55 \pm 0.87$ & $8.45 \pm 0.23$ & $6.65 \pm 0.12$ \\
\hline \multirow{2}{*}{ G. applanatum } & Gujrat & $10.35 \pm 0.62$ & $13.75 \pm 0.26$ & $13.25 \pm 0.53$ & $13.85 \pm 0.35$ & $7.15 \pm 0.19$ & $5.95 \pm 0.91$ \\
\hline & Shugran & $10.05 \pm 0.45$ & $12.15 \pm 0.53$ & $10.00 \pm 0.62$ & $15.25 \pm 0.26$ & $17.05 \pm 0.35$ & $13.35 \pm 0.54$ \\
\hline G. austral & Murree & $7.15 \pm 0.12$ & $22.45 \pm 0.21$ & $10.25 \pm 0.63$ & $5.55 \pm 0.36$ & $22.35 \pm 0.19$ & $10.65 \pm 0.91$ \\
\hline \multirow{2}{*}{ G. colossus } & Lahore & $10.45 \pm 0.17$ & $18.55 \pm 0.71$ & $11.45 \pm 0.01$ & $12.75 \pm 0.10$ & $20.85 \pm 0.52$ & $13.15 \pm 0.25$ \\
\hline & Chhanga Manga & $13.05 \pm 0.54$ & $10.95 \pm 0.65$ & $13.80 \pm 0.76$ & $12.55 \pm 0.87$ & $12.40 \pm 0.98$ & $8.05 \pm 0.10$ \\
\hline \multirow{2}{*}{ G. flexipes } & Kaghan & $13.60 \pm 0.56$ & $9.05 \pm 0.67$ & $13.70 \pm 0.78$ & $12.55 \pm 0.89$ & $12.40 \pm 0.90$ & $9.75 \pm 1.01$ \\
\hline & Murree & $15.55 \pm 0.03$ & $20.30 \pm 1.65$ & $10.55 \pm 0.34$ & $15.60 \pm 0.72$ & $5.10 \pm 0.41$ & $5.00 \pm 0.53$ \\
\hline G. resinaecum & Lahore & $6.05 \pm 1.45$ & $13.75 \pm 0.76$ & $11.55 \pm 0.32$ & $7.35 \pm 0.64$ & $9.45 \pm 1.23$ & $8.25 \pm 1.87$ \\
\hline G. tornatum & Murree & $8.35 \pm 0.76$ & $11.05 \pm 0.12$ & $12.95 \pm 0.24$ & $15.15 \pm 0.48$ & $22.85 \pm 0.96$ & $13.25 \pm 0.89$ \\
\hline
\end{tabular}


Vol. 65 (5): 2020

\begin{tabular}{|c|c|c|c|c|c|c|c|}
\hline \multirow{3}{*}{ Tramets hirsutus } & Lahore & $14.75 \pm 0.98$ & $21.00 \pm 0.76$ & $20.05 \pm 0.56$ & $11.35 \pm 0.75$ & $5.50 \pm 0.75$ & $5.55 \pm 0.46$ \\
\hline & Sialkot & $12.10 \pm 0.82$ & $17.95 \pm 0.64$ & $15.60 \pm 0.65$ & $12.30 \pm 0.56$ & $11.45 \pm 0.78$ & $9.45 \pm 0.34$ \\
\hline & Changa manga & $12.05 \pm 0.68$ & $19.00 \pm 0.73$ & $12.35 \pm 0.24$ & $12.40 \pm 0.48$ & $13.55 \pm 0.96$ & $12.25 \pm 0.69$ \\
\hline \multirow{3}{*}{ T. proteus } & Sialkot & $15.05 \pm 0.34$ & $21.50 \pm 0.68$ & $14.90 \pm 0.23$ & $10.00 \pm 0.46$ & $7.45 \pm 0.56$ & $6.80 \pm 1.12$ \\
\hline & Sangla Hill & $10.05 \pm 2.56$ & $11.50 \pm 0.98$ & $14.95 \pm 0.33$ & $7.30 \pm 0.66$ & $18.25 \pm 0.34$ & $7.05 \pm 0.68$ \\
\hline & Chhanga Manga & $5.95 \pm 0.56$ & $19.75 \pm 0.67$ & $11.35 \pm 0.45$ & $10.15 \pm 0.90$ & $11.45 \pm 0.34$ & $9.75 \pm 0.26$ \\
\hline \multirow{2}{*}{ T. pubescens } & Chhang Manga & $13.65 \pm 0.78$ & $21.40 \pm 0.67$ & $8.80 \pm 0.76$ & $7.05 \pm 0.54$ & $28.05 \pm 0.98$ & $8.50 \pm 0.32$ \\
\hline & Sialkot & $6.15 \pm 0.64$ & $19.45 \pm 0.76$ & $9.95 \pm 0.98$ & $13.70 \pm 0.54$ & $11.05 \pm 0.08$ & $8.55 \pm 0.97$ \\
\hline \multirow{2}{*}{ T. tephroleucus } & Naran & $10.35 \pm 0.98$ & $13.70 \pm 0.76$ & $12.55 \pm 0.34$ & $8.00 \pm 0.45$ & $11.45 \pm 0.76$ & $5.90 \pm 0.29$ \\
\hline & Chhangla Gully & $7.35 \pm 0.76$ & $15.50 \pm 0.54$ & $13.10 \pm 0.65$ & $10.25 \pm 0.19$ & $9.50 \pm 0.65$ & $7.55 \pm 0.62$ \\
\hline \multirow{2}{*}{ T. versicolor } & Kalam & $11.90 \pm 0.86$ & $13.45 \pm 1.35$ & $11.35 \pm 0.86$ & $14.70 \pm 0.76$ & $24.40 \pm 0.53$ & $14.85 \pm 0.98$ \\
\hline & Shugran & $7.80 \pm 0.76$ & $16.45 \pm 0.62$ & $14.90 \pm 0.74$ & $12.75 \pm 0.73$ & $13.15 \pm 0.87$ & $10.30 \pm 0.26$ \\
\hline \multirow{2}{*}{ T. zonatus } & Murree & $14.60 \pm 0.56$ & $11.20 \pm 0.67$ & $9.55 \pm 0.76$ & $10.10 \pm 2.09$ & $8.25 \pm 1.34$ & $6.50 \pm 1.67$ \\
\hline & Kalam & $12.05 \pm 0.97$ & $10.10 \pm 1.54$ & $8.25 \pm 0.86$ & $12.50 \pm 0.87$ & $21.35 \pm 0.35$ & $19.70 \pm 0.97$ \\
\hline Fomes ajazii & Murree & $9.75 \pm 0.67$ & $11.85 \pm 0.87$ & $10.80 \pm 0.54$ & $14.95 \pm 0.78$ & $22.90 \pm 0.65$ & $15.30 \pm 0.45$ \\
\hline \multirow{4}{*}{ F. borneonensis } & Kaghan & $15.60 \pm 0.67$ & $12.35 \pm 0.43$ & $9.65 \pm 0.67$ & $12.40 \pm 0.67$ & $12.70 \pm 0.23$ & $8.45 \pm 0.56$ \\
\hline & Kalam & $7.75 \pm 0.76$ & $24.50 \pm 0.34$ & $9.80 \pm 0.12$ & $11.55 \pm 0.24$ & $13.85 \pm 0.36$ & $12.60 \pm 0.48$ \\
\hline & Sawat & $10.65 \pm 0.60$ & $8.90 \pm 0.72$ & $10.70 \pm 0.84$ & $10.95 \pm 0.96$ & $14.75 \pm 1.08$ & $8.00 \pm 1.20$ \\
\hline & Kashmir, Bagh & $7.80 \pm 1.32$ & $16.05 \pm 1.44$ & $12.85 \pm 1.56$ & $5.10 \pm 1.68$ & $22.90 \pm 1.80$ & $11.15 \pm 1.92$ \\
\hline \multirow{2}{*}{ F. fomentarius } & Azad Kashmir & $11.95 \pm 1.04$ & $24.20 \pm 1.26$ & $10.00 \pm 0.14$ & $10.25 \pm 0.02$ & $11.05 \pm 0.14$ & $9.30 \pm 1.26$ \\
\hline & Abbot Abad & $6.10 \pm 1.36$ & $21.35 \pm 1.48$ & $11.15 \pm 1.60$ & $6.40 \pm 0.48$ & $10.20 \pm 0.36$ & $7.45 \pm 0.24$ \\
\hline F. Linteus & Murree & $12.25 \pm 0.56$ & $9.50 \pm 0.44$ & $7.30 \pm 0.32$ & $11.55 \pm 0.20$ & $20.35 \pm 0.08$ & $11.60 \pm 0.20$ \\
\hline \multirow{3}{*}{ F. scruposus } & Murre & $11.40 \pm 0.32$ & $17.65 \pm 0.44$ & $12.45 \pm 0.56$ & $16.70 \pm 0.68$ & $21.50 \pm 0.80$ & $14.75 \pm 0.92$ \\
\hline & Shugran & $5.55 \pm 0.13$ & $7.80 \pm 0.26$ & $10.60 \pm 0.39$ & $15.85 \pm 0.53$ & $33.65 \pm 0.66$ & $17.90 \pm 0.79$ \\
\hline & Kalam & $11.70 \pm 0.90$ & $19.95 \pm 1.05$ & $9.75 \pm 1.16$ & $9.00 \pm 0.12$ & $11.80 \pm 0.25$ & $9.05 \pm 0.37$ \\
\hline F. semitostu & Gilgit & $12.85 \pm 0.50$ & $18.10 \pm 0.76$ & $10.90 \pm 0.89$ & $8.15 \pm 0.92$ & $12.95 \pm 0.02$ & $9.20 \pm 0.02$ \\
\hline \multirow{3}{*}{$\begin{array}{c}\text { Heterobasidion } \\
\text { insulare }\end{array}$} & Nathia gully & $12.00 \pm 1.01$ & $30.25 \pm 0.27$ & $13.05 \pm 0.58$ & $14.30 \pm 0.72$ & $11.10 \pm 1.97$ & $10.35 \pm 2.01$ \\
\hline & Dunga Gully & $8.15 \pm 0.76$ & $16.40 \pm 0.59$ & $9.20 \pm 0.08$ & $11.60 \pm 0.67$ & $10.25 \pm 0.45$ & $10.45 \pm 0.59$ \\
\hline & Kaghan & $11.30 \pm 0.65$ & $12.75 \pm 0.38$ & $10.55 \pm 0.48$ & $11.85 \pm 0.49$ & $22.35 \pm 0.29$ & $20.60 \pm 0.18$ \\
\hline \multirow{2}{*}{$\begin{array}{c}\text { Poria } \\
\text { latemarginata }\end{array}$} & Faisal Abad & $9.95 \pm 0.48$ & $15.60 \pm 0.49$ & $13.40 \pm 0.49$ & $6.00 \pm 0.30$ & $8.35 \pm 0.63$ & $7.55 \pm 0.52$ \\
\hline & Chhanga Manga & $10.75 \pm 1.69$ & $7.60 \pm 0.04$ & $8.80 \pm 0.48$ & $12.65 \pm 0.05$ & $8.25 \pm 0.54$ & $9.15 \pm 0.42$ \\
\hline P. paradoxa & Chhanga Manga & $5.65 \pm 0.92$ & $6.85 \pm 0.58$ & $5.75 \pm 0.59$ & $11.60 \pm 0.03$ & $20.20 \pm 0.09$ & $15.20 \pm 0.23$ \\
\hline P. ravenalae & Lahore & $20.70 \pm .58$ & $13.80 \pm 0.21$ & $11.80 \pm 0.43$ & $10.55 \pm 0.64$ & $20.25 \pm 0.85$ & $19.45 \pm 1.07$ \\
\hline P. versipora & Chhanga Manga & $6.90 \pm 1.28$ & $7.00 \pm 1.49$ & $9.60 \pm 0.76$ & $11.75 \pm 0.23$ & $28.05 \pm 0.49$ & $24.65 \pm 0.21$ \\
\hline \multirow{2}{*}{$\begin{array}{c}\text { Phellinus } \\
\text { allardii }\end{array}$} & Murree & $10.60 \pm 0.43$ & $17.70 \pm 0.37$ & $14.30 \pm 0.53$ & $6.45 \pm \pm 0.62$ & $7.85 \pm 0.69$ & $8.35 \pm 0.65$ \\
\hline & Kaghan & $15.70 \pm 0.63$ & $16.80 \pm 0.27$ & $13.40 \pm 0.51$ & $10.55 \pm 0.63$ & $21.95 \pm 0.61$ & $19.45 \pm 0.63$ \\
\hline \multirow{3}{*}{ P. badius } & SialKot & $11.50 \pm 0.72$ & $12.40 \pm 1.45$ & $10.00 \pm 0.92$ & $6.15 \pm 1.54$ & $14.75 \pm 0.43$ & $15.20 \pm 1.65$ \\
\hline & Gujranwala & $13.20 \pm 1.21$ & $22.60 \pm 0.93$ & $12.00 \pm 0.52$ & $10.60 \pm 0.62$ & $9.30 \pm 0.53$ & $7.80 \pm 0.42$ \\
\hline & Lahore & $7.30 \pm 0.42$ & $8.70 \pm 0.76$ & $5.10 \pm 1.04$ & $5.10 \pm 0.85$ & $7.40 \pm 0.42$ & $9.90 \pm 0.31$ \\
\hline P. callimorphus & Murree & $11.25 \pm 0.63$ & $10.65 \pm 0.12$ & $11.05 \pm 0.73$ & $12.05 \pm 0.63$ & $32.35 \pm 1.73$ & $22.85 \pm 1.37$ \\
\hline \multirow{2}{*}{ P. caryophylli } & Kaghan & $9.55 \pm 0.56$ & $13.95 \pm 0.65$ & $11.35 \pm 0.78$ & $11.05 \pm 0.87$ & $7.65 \pm 0.76$ & $5.15 \pm 0.65$ \\
\hline & Chhanga Manga & $7.75 \pm 0.56$ & $12.15 \pm 0.26$ & $11.55 \pm 0.59$ & $7.25 \pm 0.81$ & $22.85 \pm 0.54$ & $19.35 \pm 0.72$ \\
\hline us & Lahore & $6.70 \pm 0.85$ & $14.10 \pm 0.31$ & $8.50 \pm 0.65$ & $12.20 \pm 0.72$ & $22.80 \pm 0.85$ & $20.30 \pm 0.72$ \\
\hline \multirow{3}{*}{ P.pini } & Murree & $11.50 \pm 0.21$ & $18.10 \pm 0.54$ & $15.30 \pm 0.87$ & $15.00 \pm 0.10$ & $13.60 \pm 0.44$ & $12.10 \pm 1.77$ \\
\hline & Kalam & $5.55 \pm 0.76$ & $9.15 \pm 0.32$ & $13.35 \pm 0.09$ & $11.05 \pm 0.52$ & $22.65 \pm 0.13$ & $19.15 \pm 0.04$ \\
\hline & Shugran & $11.25 \pm 0.08$ & $6.75 \pm 0.19$ & $9.75 \pm 0.64$ & $5.25 \pm 0.06$ & $21.35 \pm 0.65$ & $18.75 \pm 0.91$ \\
\hline \multirow{2}{*}{ P. torulosus } & Sawat & $9.0 \pm 0.65$ & $11.15 \pm 0.60$ & $11.15 \pm 0.55$ & $7.65 \pm 0.50$ & $8.55 \pm 0.45$ & $5.30 \pm 0.40$ \\
\hline & Muzaffar Abad & $5.05 \pm 0.67$ & $7.20 \pm 0.12$ & $5.20 \pm 0.05$ & $10.70 \pm 0.94$ & $7.60 \pm 0.08$ & $8.35 \pm 0.62$ \\
\hline
\end{tabular}




\begin{tabular}{|c|c|c|c|c|c|c|c|}
\hline $\begin{array}{c}\text { Schizophyllum } \\
\text { commune }\end{array}$ & Chitral & $10.10 \pm 0.74$ & $20.30 \pm 0.93$ & $14.50 \pm 0.67$ & $10.80 \pm 0.19$ & $22.75 \pm 0.56$ & $19.55 \pm 0.43$ \\
\hline \multirow{2}{*}{ S. raiatum } & Murree & $13.25 \pm 0.63$ & $17.35 \pm 0.65$ & $14.55 \pm 0.23$ & $19.85 \pm 0.15$ & $18.80 \pm 0.07$ & $16.60 \pm 0.20$ \\
\cline { 2 - 8 } & Sawat & $10.55 \pm 0.78$ & $17.65 \pm 0.14$ & $14.85 \pm 0.63$ & $14.15 \pm 0.37$ & $13.95 \pm 0.85$ & $12.85 \pm 0.72$ \\
\hline Daldinia sp. & Chhanga Manga & $12.25 \pm 0.70$ & $7.350 .81 \pm$ & $5.45 \pm 0.06$ & $10.05 \pm 0.47$ & $10.45 \pm 1.04$ & $8.45 \pm 1.21$ \\
\hline \multirow{2}{*}{ Xylaria sp. } & Sawat & $6.35 \pm 0.63$ & $17.40 \pm 0.54$ & $13.55 \pm 0.23$ & $15.10 \pm 0.64$ & $21.55 \pm 0.32$ & $14.50 \pm 0.94$ \\
\cline { 2 - 8 } & $\begin{array}{c}\text { Chhanaga } \\
\text { Manga }\end{array}$ & $11.40 \pm 0.05$ & $12.35 \pm 0.12$ & $11.60 \pm 0.31$ & $11.05 \pm 0.07$ & $22.60 \pm 0.63$ & $19.45 \pm 0.01$ \\
\hline
\end{tabular}

In current years, the potentials of using microorganisms as biological producers of industrially important enzymes have gained great interest in consideration of extracellular/intracellular enzymatic activity among several microorganisms (Oumar and Abate 2018). Enzyme production by mushrooms and filamentous fungi has been proven most efficient, cheaper, environment friendly and in most of the cases high yielding (Ghazala et al. 2016, Kumar et al. 2013, Martins et al. 2002, More et al. 2011). Wood rotting fungi have been reported as a most important source of economic losses in both timber fabrication and wood in use, and one of the major causes of the tree wind falls and branch drops. Since the biological aspects of these fungi varied, their discovery and identification are essential for their proper applications, management approaches and their control. The prelude studies involved isolation, identification and screening of potent native fungi showing high laccase enzyme synthesis, based on their hydrolytic activity expressed in the formation of hydrolytic zone around the colonies growing in petriplates against ABTS substrate. The study is analogous to the work of (Bonugli-Santos et al. 2010, Ho and Sze, 2018, Kiiskinen et al. 2004, Kumar et al. 2013, Kumari et al. 2012, Viswanath et al. 2008). The result of primary screening pointed out that $\mathrm{pH}$ of the medium plays vital role for growth and propagation of fungi, hence directly affecting their metabolic activities. The $\mathrm{pH} 5$ was spotted as suitable point for the paramount enzyme synthesis. The investigation disclosed that selected fungi are acidophilic and have potential to synthesize extracellular enzyme at low $\mathrm{pH}$. A large number of evidences are present to back up that optimal mushroom growth has been achieved in acidic media, though the variation exist in the required $\mathrm{pH}$ level according to different species and different constituents of growth media (Jeřábková an Tesařová 2018, Kaneko et al. 2009, Kim et al. 2002, Patel et al. 2009, Perumal et al. 2000).

The assortment of more competent ligninolytic strain was done on biochemical basis. As indicated by the results produced after submerged fermentation, it is clear that enzyme activities of the selected strains varied considerably from each other even they were from the same classification groups. These results are closely related with the work on screening of white rot fungi for ligninolytic activity of laccase enzyme (Bodke et al. 2012, Jebapriya and Gnanadoss 2014, Kumar et al. 2013). Risdianto et al. (2012) produced laccase through solid state fermentation after screening white rot fungi Marasmius sp, Trametes hirsuta, Trametes versicolor and Phanerochaete chrysosporium. Marasmius sp. was found to be highly potent among the selected fungi towards laccase production. Fifty six white rot mushroom samples were collected by Selvam et al. (2012), from Western Ghats areas of South India and were screened on two dyes Poly R-478 and Ramazol brilliant blue. Fu et al. (2013) also worked on diverse variety of white rot fungus to screen out positive strain for laccase activity and reported new isolated white rot fungus Psathyrella candolleana showing positive laccase activity. In another study Fen et al. (2014), screened ten mushroom species, Lentinus edodes 939, Pholiota nameko, L. edodes 868, Macrolepiota procera, Grifola frondosa, Pleurotus nebrodensis, and Shiraia bambusicola, Hericium erinaceus, Coprinus comatus, Auricularia auricula for the production of CMCase and laccase. In another study Santos 
et al. (2015) reported laccase synthesis through submerged fermentation form a group of white rot fungi named Agaricomycetes isolated from Amazon forest after quantitative screening.

Different indicator compounds, Poly R-478, Guaiacol, RBBR (remazol brilliant blue R) and tannic acid have been used by Alfarra et al. (2013), Kiiskinen et al. (2004) and Viswanath et al. (2008) and for the screening of novel laccase producing microbes. Their results indicated that guaiacol and tannic acid can be used for laccase activity assay. Their study concluded that ABTS was very useful for screening of ligninolytic fungi similar with the present work.

\section{CONCLUSIONS}

Qualitative assay analysis revealed that the all test fungi possess good ability for synthesis of laccase enzyme at $\mathrm{pH} 5.5$ on MEA (malt extract agar) media along with ABTS. Ganoderma lucidum exhibited marvelous potential towards Laccase biosynthesis $42.85 \pm 0.54 \mathrm{~mm}$ zone of inhibition followed by Pleurotus osteratus with $41.35 \pm 1.24 \mathrm{~mm}$.

\section{REFERENCES}

1. Alfarra, H.Y., Hasali, N.H.M., Omar, M.N., 2013: A lignolytic fungi with laccase activity isolated from Malaysian local environment for phytochemical transformation purposes. International Research Journal of Biological Science 2: 51-54.

2. Arora, D.S., Gill, P.K., 2012: Production of ligninolytic enzymes by Phlebia floridensis. World Journal of Microbiology and Biotechnology 21: 1021-1028.

3. Baldrian, P., 2006: Fungal laccases occurrance and properties. FEMS Microbiology Reviews 30(2): 215-242.

4. Bodke, P.M., Senthilarusa, G., Raghukumar, S., 2012: Screening diverse fungi for laccases of varying properties. Indian Journal of Microbiology 52(2):247-250.

5. Bonugli-Santos, R., Durrant, L.R., Silva, M.D., Sette, L.R., 2010: Production of laccase manganese peroxidase and lignin peroxidase by Brazilian marine derived fungi. Enzyme microbial Technology 46(1): 32-37.

6. Chefetz, B., Chen Y., Hadar, Y., 1998: Purification and characterization of laccase from Chaetomium thermophilium and its role in humification. Applied and Environmental Microbiology 64(9): 3175-3179.

7. Claus H., 2004: Laccases: structure, reactions, distribution. Micron 35(1-2): 93-96.

8. Dittmer, N.T., Gorman, M.J., Kanost M.R., 2009, Characterization of endogenous and recombinant forms of laccase-2, a multicopper oxidase from the tobacco hornworm, Manduca sexta. Insect Biochemistry Molecular Biology 39(9): 596-606.

9. Eggert, C., Temp, U., Eriksson, K.L., 1996: The ligniolytic system of white rot fungus Pycnoporus cinnabarinus: purification and characterization of laccase. Applied and environmental Microbiology 62(4): 1151-1158.

10. Fen, L., Xuwei, Z., Nanyi, L., Puyu, Z., Shuang, Z., Xue, Z., Pengju, L., Qichao, Z., Haiping, L., 2014: Screening of lignocellulose-degrading superior mushroom strains and determination of their CMCase and Laccase activity. The scientific World Journal Article ID 763108, 6 pp.

11. Fu, K., Fu, S., Zhan, H., Zhou, P., Liu, M., Liu, H., 2013: A newly isolated white rot fungus for laccase production in submerged cultures. BioResources 8(1): 1385-1397. 
12. Ghazala, I., Haddar, A., Rombhane, M.B., Chaanouni, S.E., 2016: Screening and molecular identification of new microbial strains for production of enzymes of biotechnological interest. Brazilian Archives of Biology and Technology, 59: e16150152.

13. Gianfreda, L., Xu, F., Bollag, J.M., 1999: Laccases: a useful group of oxido-reductive enzymes. Bioremediation Journal 3(1): 1-25.

14. Gonthier, P., Nicolotti, G., 2007: A field key to identify common wood decay fungal species on standing trees. Arboric Urban for 33: 410-420.

15. Ho, J.C.K., Sze, L.Y., 2018: Isolation, identification and characterization of enzyme producing lactic acid bacteria from traditional fermented foods. Bioscience Horizons: The International Journal of Student Research, 11: hzy004.

16. Hou, H.M., Zhou, J.T., Wang, J., Du, C.H., Yan, B., 2004: Enhancement of laccase production by Pleurotus ostreatus and its use for the decolorization of anthraquinone dye. Process Biochemistry 39(11): 1415-1419.

17. Jebapriya, G.R., Gnanadoss, J.J., 2014: Screening and molecular characterization of white rot fungi capable of laccase production and dye decolourization. Internatinal Journal of Life Science and Pharma Research 4(2): 12-20.

18. Jeřábková, J., Tesařová, D., 2018: Resistance of various materials and coatings used in wood constructions to growth of microorganisms. Wood Research 63(6): 993-1002.

19. Kaneko, S., Cheng, M., Murai, S., Takenaka, S., Murakami, S., Aoki, K., 2009: Purification and characterization of an extracellular laccase from Phlebia radiate strain BP-11-2 that decolorizes fungal melanin. Bioscience, Biotechnology and Biochemistry 73: 939-942.

20. Kiiskinen, L.L., Viikari, L., Kruus, K., 2004: Purification and characterisation of a novel laccase from the ascomycete Melanocarpus albomyces. Applied Microbiology Biotechnology 59: 198-204.

21. Kim, Y., Cho, N.S., Eom, T.J., Shin, W., 2002: Purification and characterization of a laccase from Cerrena unicolor and its reactivity in lignin degradation. Bulletin of Korean Chemical. Society 23: 985-989.

22. Kumar, R., Tapwal, A., Pandey, S., Borah, R.K., Borah, D., Borgohain, J., 2013: Macrofungal diversity and nutrient content of some edible mushrooms of Nagaland, India. Nusantara Bioscience 5(1):1-7.

23. Kumaran, S.S., Kavitha, C., Ramesh, M., Grumnt, T., 2011: Toxicity studies of nonylphenol and octylphenol: hormonal haematological and biochemical effects in Clarias gariepinus. Journal of Applied Toxicology 31(8): 752-761.

24. Kumari, B., Upadhyay, R.C., Artri, N.S., 2012: Screening and evaluation of extra-cellular oxidases in some termitophilous and lepiotoid mushrooms. World Journal of Agricultural Sciences 8(4): 409-414.

25. Li, L., Yu, L., Wu, Z., Hu, Y., 2019: Delignification of poplar wood with lactic acid-based deep eutectic solvents. Wood Research 64(3): 2019 499-514.

26. Luley, C.J., 2005: Wood decay fungi common to urban living trees in the northern and central United States. Urban Forestry LLC, $61 \mathrm{pp}$.

27. Lundell, T., Leonowicz, A., Rogalski, J., Hatakka, A., 1990: Formation and action of lignin modified enzymes in culture of Phelbia radiata supplemented with verataric acid. Applied Environmental Microbiology 56: 2623-2629.

28. Martins, L.O., Soares, C.M., Pereira, M.M., Teixeira, M., Costa, T., Jones, G.H., 2002: Molecular and biochemical characterization of a highly stable bacterial laccase that occurs as a structural component of the Bacillus subtilis endospore coat. Journal of Biological Chemistry 277: 18849-18859. 
29. More, S.S., Renuka, P.S., Pruthvi, K., Swetha, M., Malini, S., Veena, S.M., 2011: Isolation and purification of fungal laccases from Pleurotus ostreatus. Enzyme Research (1): 248735.

30. Oumer, O.J., Abate, D., 2018: Screening and molecular identification of pectinase producing microbes from coffee pulp. BioMed Research International, article ID 2961767, 7 pp.

31. Patel, H., Gupte, A., Gupte, S., 2009: Effect of different culture conditions and inducer on production of laccase by basidiomycete fungal isolate Pleurotus ostreatus HP-1 under solid state fermentation. BioResources 4(1): 268-284.

32. Pažitný, A., Russ, A., Bohacek, S., Stankovska, M., 2019: Various lignocellulosic raw materials pretreatment processes utilizable for increasing holocellulose accessibility for hydrolytic enzymes. Part I. Evaluation of wheat straw pretreatment processes. Wood Research 64(1): 13-24.

33. Perumal, K., Murugesan, K., Kalaichelvan, P.T., 2000: Influence of culture parameters on paper mill effluent decolorization by a white rot fungus Ganoderma lucidum. Indian Journal of Experimental Biology 38(04): 385-389.

34. Pointing, S.B., Vrijmoed, L.L.P., 2000: Decolourization of azo and triphenylmethane dyes by Pycnoporus sanguinues producing laccase as the sole phenoloxidase. World Journal of Microbiology and Biotechnology 16: 317-318.

35. Risdianto, H., Sofianti, E., Suhardi, S.H.A., Setaidi, T., 2012: Optimisation of laccase production using white rot fungi and agriculture wastes in solid state fermentation. Journal of Engineering Science 44(2): 93-105.

36. Santos, Y.V., Freire, D.A., Pinheiro, S., Fontao, L., Braga de Souza, J.V., Cavallazio, J.R.P., 2015: Production of laccase from white rot fungi isolated from the Amazon forest for oxidation of Razmol Brilliant Blue R. Scientific Research and Assays 10(4): 132-136.

37. Selvam, K., Priya, M.S., Sivaraj, C., Arungandhi, K., 2012: Identification and screening of wood rot fungi from Western Ghats area of South India. World journal of Chemtech Research 4(1): 379-388.

38. Songulashvili, G., Elisashvili, V., Wasser ,S.P., Nevo, E., Hadar, Y., 2007: Basidiomycetes laccase and manganese peroxidase activity in submerged fermentation of food industry wastes. Enzyme and Microbial Technology 41(1-2): 57-61.

39. Souza, C.G.M., Zilly, A., Peralta, R.M., 2002: Production of laccase as the sole phenoloxidase by a Brazilian strain of Pleurotus pulmonarius in solid state fermentation. Journal of Basic Microbiology 42(2): 83-90.

40. Tagger, S., Perissol, C., Gil, G., Vogt, G., Le, Petit J., 1998: Phenoloxidases of the whiterot fungus Marasmius qurcophilus isolated from an evergreen oak litter (Quercus ilex L). Enzyme Microbiology Technology 23(6): 372-379.

41. Viswanath, B., Chandra, M.S., Pallavi, H., Reddy, B.R., 2008: Screening and assessment of laccase producing fungi isolated from different environmental samples. African Journal of Biotechnology 7(8): 1129-1133.

\author{
Zill-E-Huma Aftab* \\ University of The Punjab \\ Institute of Agricultural Sciences \\ LAHORE \\ PAKISTAN
}

*Corresponding author: m.muzamilaftab@gmail.com 


\author{
Shakil Ahmed \\ University of the Punjab \\ Department of Botany \\ LAHORE \\ Pakistan \\ Arusa Aftab \\ Lahore College for Women University \\ Department of Botany \\ LAHORE \\ Pakistan \\ IfFat Siddique \\ Eastern Cereal and Oilseed Research Centre \\ OtTAWA \\ Canada \\ Muzammil Aftab \\ Government College University \\ LAHORE \\ Pakistan \\ Zubaida Yousaf \\ Lahore College for Women University \\ Department of Botany \\ LAHORE \\ Pakistan \\ Farman Ahmed Chaudhry \\ Minhaj University \\ Department of Food Science and nutrition \\ LAHORE \\ Pakistan
}

